\title{
El uso de podcast como instrumento de evaluación sobre el aprendizaje en la enseñanza de las ciencias
}

\author{
Cristina Gil Puente - Universidad de Valladolid \\ Vanessa Ortega-Quevedo - Universidad de Valladolid
}

D 0000-0001-5794-5564

(iD) 0000-0002-5742-4678

Recepción: 06.12.2021 | Aceptado: 30.12.2021

Correspondencia a través de ORCID: Cristina Gil Puente

iD 0000-0001-5794-5564

Citar: Gil, C y Ortega-Quevedo, V (2022). El uso de podcast como instrumento de evaluación sobre el aprendizaje en la enseñanza de las ciencias. REIDOCREA, 11(2), 14-27.

Área o categoría del conocimiento: Didáctica de las Ciencias Experimentales. Formación de Formadores

Resumen: En esta investigación se aborda el uso del podcast como herramienta docente innovadora para reflexionar y evaluar el proceso de enseñanza-aprendizaje en formación de formadores, dando voz al alumnado. El objetivo principal presenta una doble vertiente, por un lado, contribuir al desarrollo de competencias para el siglo XXI y por otro analizar mediante el contenido de los podcasts el aprendizaje de los estudiantes comprobando si se han llegado a conseguir los objetivos de la materia (Didáctica de las Ciencias Experimentales). La metodología empleada es cualitativa basada en la técnica de análisis de contenido cualitativo. El análisis de resultados muestra como todos los objetivos de la materia se han visto reflejados en las reflexiones de aprendizaje de los estudiantes, con especial incidencia en las temáticas relacionadas con: promover una actitud positiva frente al proceso de enseñanza de las ciencias; comprender y fomentar la reflexión y el pensamiento crítico; el diseño de materiales didácticos y la evaluación. En consecuencia, se concluye que el uso del podcast como instrumento didáctico en este contexto promueve al desarrollo de las competencias para el siglo XXI y permite vislumbrar el conjunto de los aprendizajes obtenidos por los estudiantes.

Palabra clave: Evaluación formativa

\section{The use of podcast as an assessment tool on learning in science teaching}

\begin{abstract}
This research addresses the use of podcasts as an innovative teaching tool to reflect on and evaluate the teaching-learning process in the training of trainers, giving students a voice. The main objective is twofold: on the one hand, to contribute to the development of competences for the 21 st century and, on the other, to analyse student learning through the content of the podcasts, checking whether the objectives of the subject (Didactics of Experimental Sciences) have been achieved. The methodology used is qualitative based on the qualitative content analysis technique. The analysis of the results shows how all the objectives of the subject have been reflected in the students' learning reflections, with special emphasis on the topics related to promoting a positive attitude towards the process of teaching science; understanding and encouraging reflection and critical thinking; the design of teaching materials and evaluation. Consequently, it is concluded that the use of the podcast as a teaching tool in this context promotes the development of competences for the 21 st century and provides a glimpse of the learning obtained by the students.
\end{abstract}

Keyword: Formative assessment

\section{Introducción}

La sociedad actual está caracterizada por el progreso y la globalización (OCDE, 2018). El rápido desarrollo científico-tecnológico que se ha alcanzado a lo largo de los últimos años nos ha sumergido en la era del conocimiento. "El aumento sostenido de la disponibilidad, acceso y uso de tecnologías digitales ha tenido un profundo impacto en nuestra organización social, en la forma en la que las personas aprenden, trabajan, se entretienen y comunican" (Hinostroza, 2017, p. 3). Sin embargo, ¿está la sociedad preparada para participar en esa andanza? Ya es un hecho que las Tecnologías de la Información y la Comunicación (TIC) conforman una parte importante de nuestro hacer diario y se han integrado en trabajo y educación, generando una "cultura digital" (Hinostroza, 2017). Asimismo, los avances de los ciudadanos en el desarrollo de la 
competencia mediática son notables González-Fernández, 2018 y Gutiérrez-Martín, et al., 2019.

No obstante, ¿estas son todas las competencias necesarias que adquirir? Numerosos estudios dan cuenta de la demanda de competencias para los distintos puestos de trabajo actuales y futuros está cambiando y, por ende, los integrantes de la sociedad necesitan adquirir una serie de competencias que les permitan desenvolverse de forma autónoma y crítica en esta situación (Davies et ál., 2011; Erstad \& Voogt, 2018; van Laar et al., 2017; Schleicher, 2016). En consecuencia, la educación debe dar respuesta a esta necesidad formativa desde todas las etapas educativas (Almerich et al., 2020; Manassero y Vázquez, 2020; González-Galli, 2016; Scheid, 2016) y entender que el fomento de este desarrollo competencial está relacionado y debe hacerse desde una perspectiva global. Se ha de entender la situación social y se ha de formar a los jóvenes para que comprendan y utilicen las TIC desde una perspectiva crítica que les permita comprender su potencial y entender los posibles malos usos de estas, así como aprovecharse de sus grandes beneficios.

El desarrollo de estas competencias es reconocido por la Comisión Europea (2017) o la UNESCO (2015), pues son clave para conseguir el éxito en la sociedad actual. Entendemos pues, que estas competencias para el siglo XXI son el conjunto de conocimientos transferibles al mundo real y laboral, así como la disposición para ponerlas en práctica de forma crítica y consciente (Almerich et al., 2020). Dichas competencias, en lo que concierne a la formación se dividen en competencias de alta habilidad y las competencias en TIC. De esta forma, el primer conjunto comprende las competencias en "pensamiento crítico, pensamiento creativo, aprendizaje autogestionado, resolución de problemas, la adaptabilidad, habilidades de comunicación, y las habilidades interpersonales y de trabajo en grupo" (Almerich et al., 2020 , p. 48-49). Mientras que las competencias en TIC se conforman por competencias tecnológicas, pedagógicas y éticas (Díaz-García et al., 2016).

Esta investigación presenta los resultados de una actividad realizada por los estudiantes del grado de Educación Primaria y del Programa de Estudios Conjuntos para concluir la asignatura de Didáctica de las Ciencias Experimentales en la que se refleja el desarrollo de dichas competencias. En concreto, esta propuesta se presenta como evaluación final de la asignatura y al formar parte de una titulación basada en la formación de formadores adquiere un doble significado: evaluar y reflexionar sobre lo aprendido y acontecido en el curso empleando formas de comunicación propias de la sociedad actual (podcast) y adaptando el discurso para que sea atractivo y fructífero para a un público diverso; y reflexionar sobre la propia práctica de la acción evaluadora y del uso responsable y didáctico de las TIC.

\section{La evaluación en formación de formadores}

El proceso de evaluación suele quedar ensombrecido en muchas ocasiones por la presencia de la calificación (López-Pastor y Pérez-Puello, 2017). Hay diversos modos de entender la evaluación, en este estudio se parte del concepto de evaluación formativa como elemento clave para la mejora del aprendizaje del alumnado, de la competencia docente y de los procesos de enseñanza-aprendizaje (López-Pastor y Pérez-Puello, 2017).

No obstante, la evaluación en los grados y másteres universitarios relacionados con la formación de formadores no es solo un elemento de su propio proceso de aprendizaje, sino que también es un contenido relacionado con su formación; de aquí que dejar un espacio para trabajar la evaluación con el alumnado sea más significativo en estos y 
como indica (Souto, et al., 2020) más apreciado. Los estudiantes tienen que adquirir competencias como evaluadores y entender todos los procesos de evaluación. Uno de estos procesos es la autoevaluación entendida como el proceso metacognitivo donde el discente pone en juico su propio aprendizaje/trabajo (Fraile, et al., 2020). Este procedimiento requiere la puesta en práctica de habilidades de pensamiento superior que requieren conocer los objetivos y criterios de evaluación aplicados para que los estudiantes puedan reconocer de sus éxitos y fracasos y así poder autorregular su aprendizaje.

Asimismo, desde el propio Espacio Europeo de Educación Superior se presenta un foco en la evaluación del aprendizaje universitario, ya que se precisa un cambio desde la evaluación con intenciones acreditativas, a una evaluación formativa que contemple: (1) la exploración de nuevas técnicas e instrumentos, (2) la explicitación de los aprendizajes, (3) la ampliación de los agentes intervinientes en la evaluación, (4) la exploración de la funcionalidad pedagógica de la evaluación y (5) el desarrollo de sistemas de evaluación continua que aporten evidencias del aprendizaje (Bilbao y Villa, 2019).

\section{El uso de podcast con fines didácticos}

La elaboración de un podcast educativo se basa en la grabación de un archivo sonoro con contenidos didácticos que ha sido creado a partir de un proceso de planificación (Solano y Sánchez, 2010). Se puede comparar a un programa de radio en línea al que se puede acceder en cualquier momento y desde cualquier dispositivo electrónico, también cabe la posibilidad de descargar el archivo e incluso de suscribirte a determinados canales para estar informado puntualmente de los nuevos contenidos que se van incorporando. Puede ser elaborado por un docente o por un estudiante (como el caso que se presenta), por una empresa, por una institución, etc.

Según Gabarda et al, (2019 esto nos ayudará a desarrollar el proceso de enseñanza y de aprendizaje de la manera más enriquecedora posible pues se aprovechan los recursos y medios que las tecnologías ponen a nuestro alcance.

A continuación, se enumeran algunos de los beneficios que aporta la elaboración de podcast en educación, todos ellos están directamente relacionados con las competencias a desarrollar para el siglo XXI:

- Aprender nuevos recursos: para los estudiantes, puede resultar un formato diferente y entretenido con el que aprender.

- Consolidar el aprendizaje: si el alumnado es el que genera su propio podcast en base a una temática concreta sobre la que investiga previamente, el aprendizaje resulta más significativo.

- Mejorar la competencia lingüística tanto a nivel escrito en el momento de preparar el guion, como oral en el momento de la grabación del podcast, teniendo en cuenta la premisa de que el discurso debe ser claro y comprensible.

- Potenciar la creatividad, pues se debe captar la atención del oyente y para ello es fundamental que el contenido sea atractivo.

- Fomentar la competencia digital y el uso de las nuevas tecnologías en educación a través de la utilización de programas de edición de audio.

- Desarrollar el pensamiento crítico y tener en cuenta los aspectos éticos de los recursos tecnológicos utilizados.

Además de las ventajas expuestas, hay que tener en cuenta que el podcast se realiza en grupo, por lo que se fomenta el trabajo colaborativo. También cabe destacar su 
finalidad de valorar la asignatura cursada, que permite adquirir una competencia evaluadora. Así como aprovechar los recursos y medios que las tecnologías ponen a nuestro alcance para desarrollar el proceso de enseñanza y de aprendizaje de la manera más enriquecedora posible.

\section{Objetivos o hipótesis}

El objetivo principal del estudio es presentar una actividad que promueve el desarrollo de competencias para el s XXI y comprobar cómo mediante el análisis del discurso recogido por los estudiantes en los podcasts resultantes de la actividad se puede evaluar el grado de consecución de los objetivos de la asignatura, siendo un instrumento de evaluación de esta.

\section{Contexto de implementación}

La asignatura de Didáctica de las Ciencias Experimentales pertenece al ámbito de las ciencias sociales, más concretamente de las ciencias de la educación y produce conocimiento didáctico específico sobre los sistemas de enseñanza-aprendizaje de las ciencias de la naturaleza con una doble finalidad: describir y explicar tales procesos y diseñar, desarrollar y evaluar propuestas de mejora de la educación científica. Uno de los principales retos a los que se enfrenta esta disciplina es hacer frente a las dificultades que plantea la creación y difusión de propuestas alternativas a la forma tradicional de enseñar las ciencias y al fracaso escolar que ello genera.

En el caso concreto del estudio que se presenta, la asignatura se imparte en tercer curso del Grado en Educación Primaria del Programa de Estudios Conjuntos y participan un total de 96 estudiantes divididos en 23 grupos de trabajo.

\section{Objetivos y contenidos de la asignatura}

Los objetivos de aprendizaje y los contenidos se encuentran alineados con los reflejados en la memoria de verificación de la titulación en la materia Enseñanza y Aprendizaje de las Ciencias Experimentales

\section{Métodos docentes}

El proceso de Convergencia Europea conduce a importantes cambios en la Universidad española con respecto a sus fines y a la metodología utilizada hasta el momento en la mayoría de las aulas universitarias. Se trata de proporcionar una formación universitaria en la que se integren armónicamente las competencias genéricas básicas, las competencias transversales relacionadas con la formación integral de las personas y las competencias más específicas que posibilitan una orientación profesional que permita a los titulados una integración en el mercado de trabajo. La necesidad de una docencia centrada en el aprendizaje de los alumnos conlleva cambios y adaptaciones importantes $y$, en definitiva, nuevos roles a asumir por parte de los docentes y de los alumnos.

Por todo lo anterior, y por coherencia con nuestras concepciones educativas, se propone una metodología activa, que parta de los intereses y necesidades del propio alumno, de manera que se fomente el aprendizaje autónomo y la autorregulación del futuro maestro, aspecto transcendental en el proceso de enseñanza-aprendizaje y en el desarrollo profesional. La metodología de enseñanza está centrada en ofrecer gran protagonismo al alumno, de tal forma que él construya su propio aprendizaje. El fomento de la reflexión, la búsqueda de información, la toma de decisiones y la resolución de problemas tiene especial interés a partir del aprendizaje que se pretende conseguir. 
El planteamiento didáctico por el que se opta transcurre a través de actividades con las que se trata de dar respuestas a cuestiones y problemas de carácter profesional y significativo para el futuro docente. Se pretende movilizar los conocimientos específicos sobre el aprendizaje del alumno, de manera que vaya produciéndose el deseable cambio progresivo hacia el descubrimiento de la realidad del aula de Primaria y en todas las actividades de ámbito científico. Todo ello supone una metodología que potencie la participación de los alumnos, así como las relaciones entre los compañeros y el profesor. Las clases se plantean como sesiones de trabajo para todos, con buen grado de implicación de las partes involucradas (clases magistrales participativas, prácticas, seminarios...).

El procedimiento de evaluación en la asignatura es acorde con el modelo didáctico y principios educativos, pretende ser integral, formativo, participativo y continuo. Ello implica que la evaluación se integra con el resto del proceso didáctico y el contexto educativo promoviendo mejora continuada del proceso de enseñanza-aprendizaje.

Durante todo el curso los trabajos efectuados por los alumnos son evaluados por las profesoras siguiendo los criterios prefijados e indicando al discente su nivel de logro en una rúbrica detallada. Posteriormente en el aula se comentan las observaciones generales y los errores más comunes. Las dudas individuales se aclaran en el horario de tutorías. El alumno tiene la posibilidad de mejorar los aspectos sugeridos por las docentes en un plazo de una semana, tras lo cual se realiza otra evaluación. Al final de curso, las docentes llevan a cabo una revisión final de las actividades entregadas y realizan salto a la calificación.

\section{Descripción de la actividad}

La actividad que se describe tiene como principal objetivo realizar una reflexión final sobre el proceso de aprendizaje en la asignatura de Didáctica de las Ciencias Experimentales en el curso académico 2019-20, y da respuesta a preguntas tales como, ¿de qué modo procurar que el alumnado emplee sus competencias de pensamiento superior, realice un uso pedagógico de las TIC y difunda los principales hitos en su aprendizaje? ¿cómo puedo evaluar si los objetivos de mi asignatura se han cumplido recurriendo al aprendizaje de mis alumnos?

El guion que se entrega a los estudiantes les facilita información sobre el proceso y las características que debe tener el podcast que elaboren. En primer lugar, se les pide que seleccionen información esencial sobre lo que se quiere analizar y decidan qué tipo de podcast quieren realizar, el formato debe ser original y creativo (un monólogo científico, un debate, una entrevista con expertos, análisis de material audiovisual desde la temática de la asignatura, etc.). Además, se debe asegurar la reflexión sobre el tema tratado mediante la inclusión de movimientos del pensamiento como hacer y resolver preguntas, relacionar contenidos, realizar interpretaciones o explicaciones, sintetizar bien la información importante (Ritchhart et al., 2011).

Las pautas recomendadas por las profesoras a los dicentes son las siguientes:

- Realizarse preguntas como ¿de qué forma puedo llamar la atención de la sociedad para que escuchen mi podcast?

- Duración: 15 - 30 minutos

- Es conveniente elaborar un guion antes de realizar la grabación. Este guion puede ser revisado por las profesoras.

- Consultar tutoriales facilitados en el guion.

- Emplear el programa audacity para la edición. 
Por último, las docentes seleccionan los podcasts que cumplen en mayor grado los criterios de evaluación para ser compartidos por las RRSS del Proyecto de Innovación Docente Pensamiento visible y nuevas tecnologías en Educación, en concreto en el canal de IVOOX \#PENSA_TIC Pensamiento y aprendizaje visible/TIC.

\section{Método}

El diseño de este estudio consiste en analizar el contenido de las producciones realizadas por los estudiantes de la asignatura de Didáctica de las Ciencias Experimentales. Se entregaron un total de 23 podcasts, uno por cada uno de los grupos de trabajo formados por los 96 estudiantes de la asignatura. El total de estos podcast fue transcrito para su posterior análisis.

La técnica empleada es el análisis de contenido cualitativo. Esta técnica posibilita el examen sistemático y objetivo del contendido de ciertos textos, con el fin de interpretar aquellos elementos que no son percibidos en una lectura inmediata (Amado, Costa y Crusoé, 2014). Para realizar esta técnica con rigor se debe emplear de "de manera sistemática, objetiva, replicable, valida" (Ruiz, 2003, p. 193). Esto se logra mediante la aplicación de un sistema de categorías (ver Tabla 1) elaborado a partir de los objetivos de la asignatura establecidos en el proyecto docente y un añadido que engloba las peculiaridades surgidas por la pandemia mundial. Asimismo, con el fin de aportar rigurosidad al estudio la categorización la aplican dos investigadores de forma individual por medio del software Atlas ti. 8. Tras dicha categorización se discuten las discrepancias y se crea un documento resultante que contiene los acuerdos llegados sobre la aplicación del sistema de categorías.

Tabla 1. Categorías de análisis basadas en los objetivos del proyecto docente.

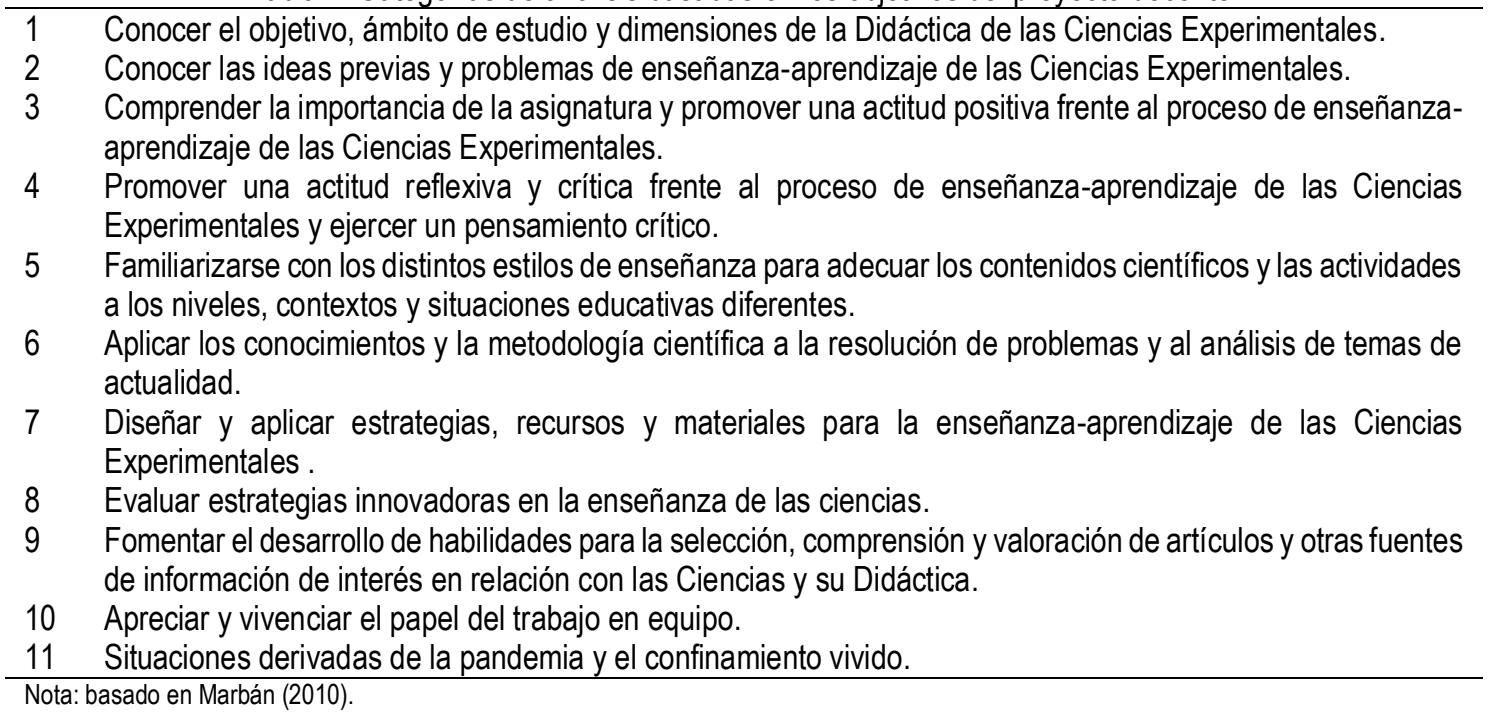

Por último, se procura preservar la confidencialidad de los estudiantes de forma que se numeran sus grupos de trabajo de forma aleatoria diferenciando entre alumnos del grado en Educación Primaria (EP) y del Programa de Estudios Conjuntos (PEC), grados en Educación Infantil y Primaria. Siguiendo estas directrices una mención al contenido del podcast realizado por los estudiantes del grupo 1 del grado de Educación Primaria se codificaría como 1EP.

\section{Resultados}




\section{Categoría 1. Conocer el objetivo, ámbito de estudio y dimensiones de la Didáctica de las Ciencias Experimentales}

En la presente categoría se han codificado 11 fragmentos de las transcripciones pertenecientes a ocho grupos. De estos fragmentos el titular que engloba todos podría ser la cita del grupo 3PEC "ciencia como patrimonio de todos al que todos tenemos acceso".

Bajo esta premisa cabe resaltar como los alumnos destacan la didáctica en la enseñanza de las ciencias en algunos fragmentos de los podcast con manifestaciones como: la importancia de conocer la forma de enseñar los distintos contenidos de la asignatura para facilitar el proceso de enseñanza-aprendizaje al alumnado, resaltan la importancia de conocer y a tender la diversidad del aula, promover la educación científica desde edades tempranas, fomentar la alfabetización científica ciudadana y entender la ciencia en el contexto próximo del alumnado.

Algunos ejemplos de dichos fragmentos son:

4PEC "lo primero que debemos saber es sobre el qué se considera importante enseñar sobre cómo aprenden los alumnos y lo más importante sobre cómo es mejor enseñar. [...] tendremos que coger contenidos que deban de aprender los niños o incluso externos [...], ver cómo aprenden mejor nuestra aula, ya que no todos los niños son iguales y entonces habrá que llevar a cabo la metodología que más se adapte a las necesidades de nuestro alumnado".

3EP "la didáctica de las ciencias experimentales lo que nos ha podido aportar es que la educación no es algo que solo se trabaja en un laboratorio con una persona que haya estudiado, sino que los niños tienen que iniciarse en ella. [...]No quiere decir que vayamos a formar científicos, sino que lo que buscamos es que entiendan la relación de su medio, de su sociedad en el contexto en el que se encuentran con la ciencia".

Por otra parte, otros fragmentos del podcast aportan un punto de vista más teórico atendiendo a los aprendizajes sobre los contenidos de naturaleza de la ciencia. Algunos ejemplos pueden ser:

10EP "la ciencia es en sí misma un fenómeno social y cultural de gran importancia construido desde hace siglos. La ciencia nos proporciona instrumentos para ser conscientes de la realidad, para pensar razonar y ser autónomo. La ciencia es un área ideal para desarrollar el pensamiento crítico del alumnado [...]. La ciencia también nos proporciona habilidades cognitivas, procedimentales, actitudinales y nos permite relacionarnos con otras personas y con el entorno."

\section{Categoría 2. Conocer las ideas previas y, errores conceptuales y problemas de enseñanza-aprendizaje de las Ciencias Experimentales}

Se ha detectado que cuatro de los 23 grupos han hablado sobre la importancia de los conocimientos previos del alumnado. En concreto se han extraído seis citas que parten del constructivismo y de la reconstrucción de los aprendizajes tomando como base los conocimientos previos de los estudiantes hasta llegar a construir conceptos más abstractos. También se destaca la importancia de averiguar los conocimientos previos de los discentes para poder adaptar el proceso de enseñanza-aprendizaje a dichos conocimientos. 
Algunos ejemplos son:

5EP "Sí, por supuesto que son importantes los aprendizajes previos. Creo que además son los que hacen de nexo de unión entre el nuevo aprendizaje y el niño, porque si nosotros conseguimos sacar los aprendizajes previos y relacionarlos con los nuevos de alguna manera están motivando al niño estamos empezando por lo que es más cercano a él, por lo que él ya sabe, por lo que le había visto, ya ha tocado y además es que las ciencias, aunque el niño no conozca terminología realmente las ciencias están en la vida del niño [...]. Por lo tanto, es fundamental que empecemos por allí."

3PEC "Es un proceso de enseñanza y aprendizaje en el que los modelos y las concepciones iniciales de los escolares generalmente alternativos a los de las ciencias, no se consideran errores, sino una etapa inicial del proceso de aprendizaje."

\section{Categoría 3. Comprender la importancia de la asignatura y promover una actitud positiva frente al proceso de enseñanza-aprendizaje de las Ciencias Experimentales}

En esta categoría se han seleccionado un total de 24 fragmentos en el discurso de 11 grupos diferentes. En esencia esos fragmentos resaltan la importancia de la enseñanza de las ciencias para comprender y poder así transmitir actitudes positivas ante las ciencias o conocimientos para poder participar y tomar decisiones relacionadas de alguna forma con la ciencia y la tecnología. Algunos señalan también como ellos mismos no tenían esta percepción de las ciencias ni de la asignatura, pero que tras el trabajo realizado durante el curso han llegado a comprender y a sentirse cómodos con esta materia.

Algunos de los fragmentos destacados son:

10EP "Si conseguimos transmitir al alumnado conocimientos y actitudes positivas de la educación científica, estos eran capaces de encontrar las reglas y las herramientas para construir un mundo más justo y sostenible. Por ello todos los alumnos independientemente de sus decisiones académicas futuras deben tener el derecho a participar en la ciencia."

7EP "bueno pues decir que la asignatura de didáctica de las ciencias experimentales, creo que me ha servido de gran ayuda para mi futuro como docente. Es un área en la que al principio no me desenvolvía muy bien [...]. Pero poco a poco, gracias a las prácticas y a los contenidos que hemos podido dar en clase y en casa he podido adquirir conocimientos nuevos [...]. Y no solo eso, sino que también hemos podido aprender cómo aplicarlos en el aula que creo que es algo fundamental. Creo que esta asignatura nos capacita como futuros docentes, algo que veo que es muy importante para poder dar clases en un futuro."

\section{Categoría 4. Promover una actitud reflexiva y crítica frente al proceso de enseñanza-aprendizaje de las Ciencias Experimentales y ejercer un pensamiento crítico}

Se ha detectado que 13 de los 23 grupos remarcan la importancia del desarrollo del pensamiento crítico y de la reflexión en el proceso de enseñanza-aprendizaje de las ciencias. En total se han codificado 20 fragmentos de las trascripciones que hace 
referencia esta temática. Si tuviéramos que elegir un titular que agrupe todos estos fragmentos podría ser el expuesto por 10EP "Pensar y razonar nos ayuda a entender el mundo y poder tomar nuestras propias decisiones". En esta línea los alumnos no solo han comprendido y valorado la necesidad de desarrollar estas habilidades de pensamiento en sus futuros alumnos, sino que han identificado sus propias carencias en el desarrollo de las mismas y agradecido la oportunidad de trabajar en la asignatura potenciando su desarrollo.

Algunos ejemplos de estas ideas son:

2EP "Uno de los puntos que más destacó ha sido como hemos aprendido a enfocar todos los trabajos y prácticas desde un enfoque crítico y aplicando las capacidades del pensamiento científico. En todas las prácticas al exigir o aplicar estas capacidades cosa que anteriormente no lo habíamos trabajado de esta manera y creo que ha sido un punto extra para potenciar capacidades, o el desarrollo de estas capacidades en nosotros."

4PEC "El alumnado recibe gran cantidad de información de diferentes medios de comunicación y de redes sociales. Estas están cargadas, en numerosas ocasiones, de intereses económicos, políticos e ideológicos, por ello tenemos que conseguir que los alumnos sean capaces de elegir aquella información que le es relevante para su aprendizaje. De esta forma el alumnado adquirirá las competencias necesarias para logar un aprendizaje significativo a través del razonamiento.

\section{Categoría 5. Familiarizarse con los distintos estilos de enseñanza para adecuar los contenidos científicos y las actividades a los niveles, contextos y situaciones educativas diferentes}

En esta categoría seleccionamos 13 extractos de las transcripciones de un total de nueve grupos. El discurso de estos fragmentos se orienta a reflexiones sobre los distintos estilos de enseñanza que se han aplicado en la asignatura cursada a modo de ejemplo de metodologías para aplicar en su futura profesión o como comentarios sobre los principales modelos de enseñanza, sus fortalezas, debilidades y cómo los llevarían a las aulas en su futuro como docentes.

Primeramente, se presentan ejemplos sobre las reflexiones relacionadas con la metodología aplicada en la asignatura cursada:

2EP "lo que más me ha gustado de todo esto haya sido que las clases han sido teóricoprácticas y a la vez quedamos la teoría se realizan prácticas de manera simultánea, con la finalidad de afianzar todos los contenidos del modo de deseado".

8PEC "yo he aplicado algunos de los métodos que ellas mismas aplicaban con nosotras, como la realización del debate sobre los modelos didácticos".

Finalmente, se recogen ejemplos sobre modelos trabajados aplicados a la enseñanza en educación infantil y primaria:

3EP "hay una serie de metodologías se podemos seguir la transmisión recepción, el modelo por descubrimiento, el constructivismo y nosotros nuestro grupo hemos querido dejar claro que no hay una metodología que puramente valga para la enseñanza ya sea de las ciencias o de cualquier otra asignatura que valga". (1:29) 
4EP "los recursos y herramientas necesarios a los alumnos para que puedan ellos mismos construir tu propio tu propio aprendizaje y esta asignatura nos ha valido mucho para eso. Hemos podido conocer multitud de recursos que podríamos llevarlos a cabo: cómo que aprendan por proyectos que realicen, experimentos en los que se pueda ver el funcionamiento de las cosas, sobre todo de su entorno".

\section{Categoría 6. Aplicar los conocimientos y la metodología científica a la resolución de problemas y al análisis de temas de actualidad}

Se han encontrado 13 fragmentos de las transcripciones de un total de ocho grupos, relacionadas con la aplicación de la metodología científica para resolver problemas de actualidad. Los principales comentarios sobre esta temática resaltan cuestiones como la aplicación de metodología científica en prácticas o la implementación de esta en contextos escolares.

1EP "la primera práctica que tuvimos que realizar utilizamos el método científico para demostrar que el aire era materia. [...] ¿te acuerdas de que ninguno descubrimos uno de los métodos, que era el poner el agua ¿verdad? Que no sabíamos descubrirlo. Todos esto es importante lo que estamos hablando porque es una forma de enseñar al alumnado, lo qué es y que puedan ver lo que usan en su día a día y no se dan cuenta de los diferentes usos que tiene".

10EP "El proceso de enseñanza de las ciencias debe de basarse la experimentación y la aplicación del método científico para comprobar las diferentes ideas que tienen los alumnos sobre los distintos fenómenos que les rodean y si estas son ciertas o no atendiendo las diferentes hipótesis propuestas.

\section{Categoría 7. Diseñar y aplicar estrategias, recursos y materiales para la enseñanza-aprendizaje de las Ciencias Experimentales con los contenidos científicos dirigidos a los distintos niveles de Educación Primaria}

En relación con la temática de esta categoría se extraen 20 citas de los podcasts de 11 grupos. Estos fragmentos hacen referencia a materiales elaborados por los alumnos durante las prácticas propuestas desde la reflexión de la utilidad que entienden que tienen los mismos o incluso a los tipos de actividades según su finalidad. Algunos de los ejemplos más representativos son:

2EP "si me tuviera que quedar con alguna de las prácticas realizadas me quedo con la del juego, ya que gracias a esta he aprendido a que se pueden enseñarlos contenidos esta materia de un modo mucho más lúdico, lo cual motivará a los alumnos".

4EP "la práctica del huerto creo que una de nuestras obligaciones como maestros debe ser conocer intentar poner en práctica cualquier tipo de estrategia o de recurso que favorezca nuestros alumnos y gracias a esta práctica nos hemos dado cuenta de que el huerto como recurso didáctico ofrece muchos beneficios para nuestros alumnos".

1PEC "Los laboratorios son un espacio algo peligroso si no se cumplen las normas o no se cuida el material que hay en él, pero que, si se dedican unas horas a familiarizar al alumnado tanto con el material, como con el espacio y las normas, puede ser un lugar lo suficientemente seguro, donde poder llevar a cabo experimentos. Además, creo que estas experiencias o experimentos que se realizan les va a servir de apoyo y refuerzo los contenidos que puedan resultar más difíciles de comprender". 


\section{Categoría 8. Evaluar estrategias innovadoras en la enseñanza de las ciencias}

En esta categoría se identifican 15 extractos de los podcasts en los que los estudiantes hablan de la evaluación. En este caso tenemos un titular sobre evaluación como cita 12EP "más que una nota, la evaluación nos permite conocer el camino adecuado y mejorar". Esta cita se extrae de una referencia que hicieron en uno de los podcasts a una actividad realizada durante la asignatura, la rutina del titular de Ritchhart (2011). Bajo este mantra el resto de los fragmentos presentan reflexiones sobre la importancia de la evaluación, los tipos, algunos instrumentos de evaluación o el sistema de evaluación llevado a cabo durante la asignatura.

Algunos ejemplos pueden ser:

9EP "tenemos un concepto demasiado tradicional acerca de la evaluación. Que simplemente es poner una nota y ya está. Yo creo que es algo que debería ir mucho más allá. Entonces dentro de lo que hemos tratado lo largo de este curso en la asignatura de Didáctica de las Ciencias Experimentales relacionándolo con el concepto de evaluación, dentro de esta misma asignatura, creo que es muy importante para poder llevar a cabo más adelante, a lo largo de nuestra labor como docentes, un proceso de evaluación correcto creo que es muy importante tener claro diferentes contextos".

5PEC "Es necesario evaluar el proceso de aprendizaje de los alumnos, está claro y la práctica docente. Realmente la evaluación es una parte la acción educativa y hay que tenerlo en cuenta. Pienso que es importante que recojamos la información durante los procesos y los distintos resultados de las actividades realizadas por los alumnos, para poder interpretarla y tomar decisiones. Debemos considerar la evaluación como un elemento para mejorar y seguir avanzando o incidir en aquellos aprendizajes que no se han alcanzado. Nos permite seguir los procedimientos realizados por los alumnos, para controlar la evolución de esos aprendizajes y mejorar el proceso de enseñanza y por lo tanto la calidad de la misma".

\section{Categoría 9. Fomentar el desarrollo de habilidades para la selección, comprensión y valoración de artículos y otras fuentes de información de interés en innovación e investigación educativa en relación con las Ciencias y su Didáctica}

Esta categoría ha sido la temática menos comentada por los estudiantes. Solamente cuatro de los grupos han hecho referencia a habilidades para la selección de fuentes de información entendiendo esta como fuentes de interés para el desarrollo de su futura profesión. Estos grupos hacen referencia a una práctica en la que se comparaban distintos libros de texto del área de ciencias de la naturaleza de distintas editoriales. Dos ejemplos que engloban el conjunto de comentarios son:

2EP "Desde el punto de vista de las prácticas realizadas personalmente quiero destacar la que hicimos comparando libros de texto de ciencias naturales, ya que se pudo ver y analizar aquello que pensábamos que le faltaba cada libro y cómo podría mejorarse, además de ver cada aspecto que tenía uno sí y otro no".

4EP "Entonces claro, según el camino que el docente quiera seguir para formar ciudadanos críticos y reflexivos pues debe escoger una editorial u otra. Sí que es verdad que hay veces que hay razones de tipo económicas que hace que un centro se decante más por una editorial o por otra. No obstante, creo que el docente debe analizarlo bien, en profundidad, y para saber cuál es el libro más adecuado y qué más se adapta pues a él y a los alumnos". 


\section{Categoría 10. Apreciar y vivenciar el papel del trabajo en equipo}

La temática relacionada con esta categoría ha sido la segunda menos citada en los trabajos. Solamente 4 grupos con un total de 5 fragmentos hablan del trabajo en equipo. Dos de los grupos hablan sobre su experiencia trabajando en grupo durante el desarrollo de la asignatura.

4PEC destaca el valor del trabajo cooperativo durante las dinámicas realizadas en el aula "Además hemos disfrutado mucho de los momentos de aprendizaje cooperativo y constructivista que se han dado en las dinámicas de clase, creemos necesario recomendaros desde aquí que los futuros cursantes de estás asignatura lleguéis con la mente abierta y con muchas ganas de aprender". Mientras que 2EP advierte la dificultad añadida de mantener el trabajo cooperativo desde que se estableció el estado de alarma: "El hecho de trabajar en grupo, pero a distancia ha supuesto una dificultad añadida para todo esto, ya que estábamos acostumbrados a vernos y a solucionar los problemas en persona, creo que esto ha supuesto también una dificultad ya que no todos podíamos trabajar online a la misma hora".

Otros estudiantes resaltan el valor del trabajo cooperativo en el contexto educativo como futuros docentes:

3EP "La enseñanza de las ciencias tiene que ser por tanto activa y cooperativa, que implique mucho al alumnado y que, como hemo dicho, el docente está allí de forma de guía".

\section{Categoría 11. Situaciones derivadas de la pandemia y el confinamiento vivido}

Por último, se reflejan los fragmentos que mencionan aspectos relacionados con la situación educativa derivada de la pandemia mundial. Respecto a esta temática los estudiantes realizan comentarios desde su propia vivencia como estudiantes de la asignatura, como estudiantes de la universidad y como futuros docentes. En total se han detectado 20 citas de un total de nueve grupos que se dividen en dos.

Algunos grupos en sus podcasts manifiesta preocupaciones sobre el desarrollo de la docencia en educación primaria como:

7PEC "Siempre se ha hablado de una educación online, pero en ningún caso se ha trabajado en ellos y de repente su implantación se ha convertido en necesario de una manera rápida y sin preparación alguna. [...] por lo que estamos perdiendo aprendizajes. Nos estamos dando cuenta de lo importante que es la presencialidad y la cercanía del docente" También dentro de este grupo se apunta que "la educación online ha sido previamente estudiada y preparada, pero la implantación de esta modalidad online creemos que llevara consigo una gran diferencia social, ya que no todos los ciudadanos tenemos el nivel económico para conseguir estos recursos tecnológicos".

14EP "Pues esperamos que esta situación nos haga más humanos y más personas, más empáticos y que eso también se vea reflejada en las aulas, pero también somos realistas y esta situación va a cambiar a todos y las medidas que se han planteado nos parecen adecuadas. Como, por ejemplo, de reducir la ratio de las aulas, pero no sabemos si en la práctica podrá llegar a ser posible debido a la falta de recursos". 
Finalmente, en relación con lo vivenciado durante la asignatura los estudiantes destacan que el cambio de modalidad en la docencia no ha sido muy determinante y se ha conseguido mantener el ritmo de aprendizaje como:

2EP "Es de agradecer la implicación por parte tanto de profesoras como de compañeros para sacar los trabajos adelante y aprender todos dentro de esta situación. Ninguno nos esperábamos esta nueva situación y como digo hemos tenido todos que trabajar para afrontarla de la manera más llevadera posible y más fructífera [...] personalmente no he notado una gran diferencia entre online y las presenciales creo que se ha hecho un gran esfuerzo por parte delas profesoras para mantener el ritmo habitual de la clase, sí que es cierto que se nota el no estás presencialmente en una clase online no se resuelven las dudas de igual manera y es más complicado el seguimiento de ritmo habitual. Aun así, creo que el desarrollo de las clases de ha sido muy parecido al que veníamos siguiendo."

Mientras otros han echado en falta cuestiones como, las salidas didácticas que había programadas, la realización de prácticas que requerían presencialidad o la interacción con sus compañeros y con las docentes de forma presencial.

6EP "nos hemos parado a pensar en todo lo que hemos perdido a causa de esta situación, como la salida al MUNCYT [...] esas clases teóricas tan dinámicas [...] esta forma de dar clase de la que tantos profesores nos hablan, pero para que la llevemos a cabo nosotros en nuestra labor docente. Nos hemos perdido también charlas de expertos, las exposiciones de las elaboraciones de experimentos del resto de la clase [...] la interacción con el resto de los compañeros".

\section{Conclusiones}

Los estudiantes mediante la aplicación de esta actividad han reflexionado sobre su aprendizaje en la asignatura. Cada uno podía elegir sobre qué tema tratar, pero los temas más recurridos han sido los correspondientes a las categorías $3,4,7$ y 8 (perspectiva positiva de la ciencia, desarrollo de habilidades y actitudes de pensamiento superior; el desarrollo de materiales didácticos en base a distintos modelos y abarcando distintos contenidos; y la evaluación).

Una de estas temáticas está directamente vinculada con las competencias para el siglo $\mathrm{XXI}$ que hemos mencionado. El alumnado no solo se da cuenta de sus propias carencias en el desarrollo de su pensamiento superior, sino que advierte la necesidad de fomentar su desarrollo en sus futuros estudiantes y encuentra en la asignatura de ciencias de la naturaleza un escenario perfecto para la enseñanza de una ciencia en contexto basada en la reflexión y las metodologías activas. Asimismo, la implementación de esta práctica ha permitido que los futuros docentes consigan hacer un uso adecuado de las TIC para plasmar sus reflexiones en un producto mediático que está disponible para aquellos interesados en escuchar programas sobre educación. Otra de las grandes vertientes a trabajar para el desarrollo de estas competencias tan necesarias.

Finalmente, el análisis de las transcripciones permite hacer un recorrido bastante completo de lo que ha sido el proceso de enseñanza-aprendizaje de la asignatura de Didáctica de las Ciencias Experimentales, dejando ver cómo las docentes han promovido una serie de aprendizajes en los alumnos que hacen ver cumplidos los objetivos del proyecto docente de la propia asignatura, a pesar de las dificultades añadidas por el cambio a la modalidad online durante el periodo de confinamiento. Sobre las características especiales de este proceso hay que destacar todas las actividades 
que han echado en falta los estudiantes en su formación, como son las salidas didácticas o las actividades de laboratorio. Además, se destaca la metodología y la proximidad del docente, aun en la distancia, como formas de mantener el ritmo de clase.

\section{Referencias}

Almerich, G, Suárez-Rodríguez, J, Díaz-García, I y Orellana, N (2020). Estructura de las competencias del siglo XXI en alumnado del ámbito educativo. Factores personales influyentes. Educación XX1, 23(1), 45-74. https://doi.org/10.5944/educXX1.23853

Amado, J, Costa, AP y Crusoé, N (2014). A técnica da análise de conteúdo. En J. Amado (Coord.), Manual de Investigação Qualitativa em Educação (pp. 301-352). Universidade de Coimbra.

Bilbao, A y Villa, A (2019). Avances y limitaciones en la evaluación del aprendizaje a partir del proceso de convergencia. Visión docente y discente en los grados de Educación Infantil y Primaria. Educación XX1, 22(1), 45-69, doi: https://doi.org/10.5944/educXX1.19976

Comisión Europea (2017). Comunicación de la Comisión al Parlamento Europeo, al Consejo, al Comité Económico y Social Europeo y al Comité de las Regiones sobre una agenda renovada de la UE para la educación superior.

Davies, A, Fidler, D, \& Gorbis, M (2011). Future Work Skills 2020. University of Phoenix Research Institute.

Díaz-García, Isabel; Cebrián-Cifuentes, Sara \& Fuster-Palacios, Isabel (2016). Las competencias en TIC de estudiantes universitarios del ámbito de la educación y su relación con las estrategias de aprendizaje. RELIEVE, 22 (1). https://dx.doi.org/0.7203/relieve.22.1.8159

Erstad, O, \& Voogt, J (2018). The twenty-first century curriculum: issues and challenges. In J Voogt, G Knezek, R Christensen, \& KW Lai (Eds.), Second Handbook of Information Technology in Primary and Secondary Education, (19-36). Springer.

Fraile, J, Gil-Izquierdo, M, Zamorano-Sande, D y Sánchez-Iglesias, I (2020). Autorregulación del aprendizaje y procesos de evaluación formativa en los trabajos en grupo. RELIEVE, 26(1). https://doi.org/10.7203/relieve.26.1.17402

Gabarda, V, Colomo, E y Romero, MM (2019). Metodologías didácticas para el aprendizaje en línea. REIDOCREA, 8(2), 19-36. https://doi.org/10.30827/Digibug.66304

González-Galli, L (2016). El problema de la teleología y la metáfora del diseño en biología: cuestiones epistemológicas e implicancias didácticas. Tecné Episteme y Didaxis: Ted, 40, 149-173. https://doi.org/10.17227/01203916.6151

González-Fernández, N, Ramírez-García, A y Salcines-Talledo, I (2018). Competencia mediática y necesidades de alfabetización audiovisual de docentes y familias españolas. Educación XX1, 21(2), 301-321. https://dx.doi.org/10.5944/educXX1.16384

Gutiérrez-Martín, A, Torrego-González, A, \& Vicente-Mariño, M (2019) Media education with the monetization of YouTube: the loss of truth as an exchange value. Cultura y Educación, 31 (2), 267-295. https://doi.org/10.1080/11356405.2019.1597443

Hinostroza, JE (2017). TIC, educación y desarrollo social en América Latina y el Caribe. UNESCO.
López-Pastor VM y Pérez-Pueyo, A (coords.) (2017). Buenas prácticas docentes. Evaluación formativa y compartida en educación: experiencias de éxito en todas las etapas educativas. León: Universidad de León.

Manassero-Mas, MA y Vázquez-Alonso, A (2020). Evaluación de destrezas de pensamiento crítico: Validación de instrumentos libres de cultura. Tecné, Episteme y Didaxis: ted, (47), 15-32. https://doi.org/10.17227/ted.num47-9801

Marbán, JM (2010). Memoria de plan de estudios del título de Grado Maestro o Maestra en Educación Primaria. Valladolid: Universidad de Valladolid.

OCDE (2018). The future of education and skills. Education 2030.

Ritchhart, R, Church, M, y Morrison, K (2011). Making Thinking Visible. How to Promote Engagement Understanding, and Independence for All Learners. Wiley.

Scheid, NMJ (2016). Os desafios da docência em ciências naturais no século xxi. Tecné, Episteme y Didaxis: ted, 40, 177-196. https://doi.org/10.17227/01203916.6153

Schleicher, A (2016). Desafíos para PISA. RELIEVE, 22(1).

Solano IM y Sánchez MM (2010). Aprendiendo en cualquier lugar: el podcast educativo. Pixel-Bit. Revista de Medios y Educación, 36 , 125-139.

Souto, R, Jiménez, F y Navarro, V (2020). Percepción de los Estudiantes sobre los Sistemas de Evaluación Formativa Aplicados en la Educación Superior. Revista Iberoamericana de Evaluación Educativa, 13(1), 11-39. https://doi.org/10.15366/riee2020.13.1.001

UNESCO (2015). Replantear la educación. ¿Hacia un bien común mundial? Paris, France: UNESCO. 